EUROPEAN JOURNAL OF PURE AND APPLIED MATHEMATICS

Vol. 13, No. 1, 2020, 84-95

ISSN 1307-5543 - www.ejpam.com

Published by New York Business Global

\title{
A Systematic Approach to Group Properties Using its Geometric Structure
}

\author{
Muhammed Bello ${ }^{1,2, *}$, Nor Muhainiah Mohd Ali ${ }^{2}$, Nurfarah Zulkifli ${ }^{2}$ \\ 1 Department of Mathematics and Computer Science, Federal University of Kashere, \\ P.M.B 0182 Gombe, Gombe State Nigeria, \\ 2 Department of Mathematical Sciences, Faculty of Science Universiti Teknologi Malaysia \\ 81310 UTM Johor Bahru, Johor
}

\begin{abstract}
The algebraic properties of a group can be explored through the relationship among its elements. In this paper, we define the graph that establishes a systematic relationship among the group elements. Let $G$ be a finite group, the order product prime graph of a group $G$, is a graph having the elements of $G$ as its vertices and two vertices are adjacent if and only if the product of their order is a prime power. We give the general presentation for the graph on dihedral groups and cyclic groups and classify finite dihedral groups and cyclic groups in terms of the order product prime graph as one of connected, complete, regular and planar. We also obtained some invariants of the graph such as its diameter, girth, independent number and the clique number. Furthermore, we used the vertex-cut of the graph in determining the nilpotency status of dihedral group. The graph on dihedral group is proven to be regular and complete only if the degree of the corresponding group is even prime power and connected for all prime power degree. It is also proven on cyclic group to be both regular, complete and connected if the group has prime power order. Additionally, the result turn out to show that any dihedral group whose order product prime graph's vertex-cut is greater than one is nilpotent. We also show that the order product prime graph is planar only when the degree of the group is three for dihedral group and less than five for cyclic group. Our final result shows that the order product prime graphs of any two isomorphic groups are isomophic.
\end{abstract}

2020 Mathematics Subject Classifications: 05C25, 20F65

Key Words and Phrases: Order product prime graph, Vertex adjacency, Graph invariant, Nilpotency of a group

\section{Introduction}

Various techniques have been used by researchers in investigating the properties of a group as well as classifying it according to its properties, which happen to be one of the

${ }^{*}$ Corresponding author.

DOI: https://doi.org/10.29020/nybg.ejpam.v13i1.3587

Email addresses: bello.m@graduate.utm.my (M. Bello), normuhainiah@utm.my (N. M. M. Ali), nurfarah3@graduate.utm.my (N. Zulkifli) 
great achievements of modern mathematics. One of the techniques found to be useful is by defining graph to the groups and investigate its properties using the corresponding geometric structure. According to Selva and Subajini [8] in order to get a better understanding of a given algebraic structure $A$, one can associate to it a graph $\Gamma$ and study the interplay of the algebraic properties of $A$ and the combinatorial properties of $\Gamma$. This study gained the attention of many researchers, for instance, Cayley graph was defined by Arthur in [2] and this graph has been used by Kelarev et al. in [7] to classify data which can be recorded as set of strings or sequences of letters over a finite alphabet. Ganesan [4] used this graph to obtain the structural description of the automorphism group of the modified bubble-sort graph. Several researches has also been conducted on the commuting graph and non-commuting graph, for instance, Sharafdini and Darbandi [10] computed the energy of commuting graph for groups whose centralizers are abelian, and in [11] Vahidi and Talebi obtained some invariants of non-commuting graphs on dihedral groups and generalized quaternion groups, which include its independent number, clique number and minimum size of the vertex cover of the graph, in their paper, they did not find the chromatic number of this graph, which has been obtained a year later by Tamizh et al. [9]. Akbari and Reza [3] used the non-commuting graph to determined up to isomophism the structure of finite non-abelian groups in which the vertex of the graph can be partitioned into two sets such that the induced subgraph on one of them is a complete graph and the induced subgraph on the other is an independent set. Ghobani and Alkhansari [5] used the geometric properties of a group to prove that for a group $G, G / Z(G) \cong \mathbb{Z}_{n} \times \mathbb{Z}_{n}$ if and only if the non-commuting graph $\Gamma(G)$ is a regular $(p+1)$-partite graph and consider the isomorphism of the non-commuting graph with some special graphs. The generalization of non-commuting graph of a group was given by Kakeri et al. in [6], where they investigated the connectivity, regularity and planarity of the graph and concurrently, give the numerical invariants of the graph which happen to be the improvement of the result given for non-commuting graphs. Abd Rani et al. [1] defined the relative coprime graph of a group with respect to its subgroups and dertermined some invariants of the graph which include the domination number, independence number and give the situation when the graph is regular. In this paper, we defined order product prime graph of finite groups, classify groups in terms of the properties of the graph as one of connected, complete, regular, planar and obtained some of its invariants such as independent number, clique number, girth and diameter. Moreover, we investigate the nilpotency status of dihedral group using the connectivity for the order product prime graphs on the dihedral group.

\section{Notations and preliminaries}

In this section, we give some basic concepts, notations and preliminaries useful to this paper.

All groups considered in this paper are finite and the investigation covered all dihedral groups $D_{n}=\left\{a, b \mid a^{n}=b^{2}=(a b)^{2}=e\right\}$ and cyclic groups $\mathbb{Z}_{n}=<g>\ni g \in \mathbb{Z}_{n}$. We denoted the identity of a group $G$ by $e$. 
On the other hand, we consider simple undirected graphs without loop or multiple edges. The sets of vertices and edges of a graph $\Gamma$ are denoted by $V(\Gamma)$ and $E(\Gamma)$ respectively. We denote the adjacency of vertices $x, y$ by $x \sim y$, number of vertices of the graph $\Gamma$ by $|V(\Gamma)|$, the degree of the vertex $v$ by $\operatorname{deg}(v)$. A graph $\Gamma$ is regular if all the vertices of the graph have the same degree, that is if for all vertices $v_{1}, v_{2}, \ldots, v_{n}$ of $\Gamma$, $\operatorname{deg}\left(v_{1}\right)=\operatorname{deg}\left(v_{2}\right)=\ldots=\operatorname{deg}\left(v_{n}\right)$ and a graph $\Gamma$ is $r$-regular if $\operatorname{deg}\left(v_{i}\right)=r, i \in \mathbb{N}$. A graph $\Gamma$ is connected if there is a path between every pair of its vertices, and a graph is complete if there is an edge between every pair of its vertices. A graph is planar if it can be drawn in a plane without edge crossing. A clique is a subset $U$ of vertices of $\Gamma$ such that the induced subgraph of $U$ is a complete graph, the size of the maximum such clique is refered to as the clique number of $\Gamma$ and denoted by $\omega(\Gamma)$. An independent set of vertices of a graph is the set of vertices such that no two vertices are adjacent, and the independent number of a graph $\Gamma$, is the cardinality of the largest independent set, which is denoted by $\alpha(\Gamma)$. The girth of a graph $\Gamma$, is the length of the shortest cycle contained in the graph, which is $\infty$ if $\Gamma$ has no cycle. The diameter of a graph is the maximum distance between the pair of its vertices, which is $\infty$ if the graph is disconnected.

Throughout this paper $p$ denotes prime number, and $\alpha \in \mathbb{N}$.

\section{Results and Discussions}

In this section, we give the formal definition of the order product prime graph and the general presentation for its connectivity, completeness, regularity and planarity on dihedral group and cyclic group, which help in obtaining its diameter, girth, independent number, and the clique number. We also use the graph properties to obtain the corresponding group properties.

The formal definition of the order product prime graph is given in Definition 1 below, followed by an example which demonstrate the definition.

Definition 1. Let $G$ be a finite group, the order product prime graph of $G$, $\Gamma^{o p p}(G)$ is a graph whose vertices are the elements of $G$ and two vertices $x, y$ are adjacent if and only if $|x||y|=p^{\alpha}, \alpha \in \mathbb{N}$, for some prime $p$.

Example 1. Consider the dihedral group of degree three which is $D_{3}=\left\{e, a, a^{2}, b, a b, a^{2} b\right\}$. $|e|=1,|a|=\left|a^{2}\right|=3,|b|=|a b|=\left|a^{2} b\right|=2$, then, there are two cliques which are the rotations $\left\{a, a^{2}\right\}$ and the reflections $\left\{b, a b, a^{2} b\right\}$, all adjacent to e. Therefore $\Gamma^{o p p}\left(D_{3}\right)=$ $K_{1}+\left(K_{2} \cup K_{3}\right)$ and is given in Figure 1 


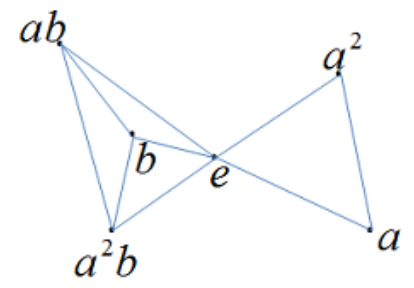

Figure 1: Order product prime graph of $D_{3}$

The general presentation for the order product prime graph on dihedral group and cyclic group are given in Theorem 1 to Theorem 3.

Theorem 1. Let $G$ be the dihedral group, $D_{n}$, where $n=p^{\alpha}, \alpha \in \mathbb{N}$, then

$$
\Gamma^{o p p}(G)=\left\{\begin{array}{l}
K_{2 n} \text { if } p=2, \\
K_{1}+\left(K_{n-1} \cup K_{n}\right) \text { if } p \neq 2 .
\end{array}\right.
$$

Proof: If $p=2$, then $|G|=2^{\alpha+1}$, pick arbitrary $x_{i}, y_{j} \in G, i \neq j$, then $|x| / 2^{\alpha+1},|y| / 2^{\alpha+1}$, that is $|x||y|=2^{t}, 1 \leq t \leq \alpha$. Since any element of $G$ is of order $2^{t}$, it follows that all the elements of $G$ form single clique. Therefore $\Gamma^{o p p}(G)=K_{2^{\alpha+1}}=K_{2 n}$.

If $p \neq 2$, then $|G|=2 p^{\alpha}$. Let $A$ and $B$ are the sets of non-trivial rotations and the reflections of $G$ respectively. pick $x \in A, y \in B$, then $x^{p^{t}}=e=y^{2}$. Now by the vertex adjacency, $A$ and $B$ are distinct cliques in $\Gamma^{o p p}(G)$ and $|(A)|=p^{\alpha}-1,|(B)|=p^{\alpha}$, then $\Gamma^{o p p}(A)=K_{p^{\alpha}-1}, \Gamma^{o p p}(B)=K_{p^{\alpha}}$ are subgraphs of $\Gamma^{o p p}(G)$. So by the vertex adjacency, $a \sim e \sim b$, therefore

$$
\begin{aligned}
\Gamma^{o p p}(G)=\Gamma^{o p p}(e)+\left(\Gamma^{o p p}(A) \cup \Gamma^{o p p}(B)\right) & =K_{1}+\left(K_{p^{\alpha}-1} \cup K_{p^{\alpha}}\right) \\
& =k_{1}+\left(K_{n-1} \cup K_{n}\right)
\end{aligned}
$$

Theorem 2. Let $G$ be a dihedral group, $D_{n}$, where $n=\prod_{i=1}^{d} p_{i}^{\alpha_{i}}$, then

$$
\Gamma^{o p p}(G)=\left\{\begin{array}{l}
\left.K_{1}+\left[\bigcup_{i=2}^{d} K_{\left(p_{i}^{\alpha_{i}}-1\right)} \cup K_{n+p_{1}^{\alpha_{1}}-1}\right)\right] \cup \bar{K}_{n+d-\left(1+\sum_{i=1}^{d} p_{i}^{\alpha_{i}}\right)} \text { where } p_{1}=2, \\
K_{1}+\left[\bigcup_{i=1}^{d} K_{\left(p_{i}^{\alpha_{i}}-1\right)} \cup K_{n}\right] \cup \bar{K}_{n+d-\left(1+\sum_{i=1}^{d} p_{i}^{\alpha_{i}}\right)} \text { if } n \text { is odd, }
\end{array}\right.
$$

where $d$ is the number of prime divisors of $n$ and $\alpha \in \mathbb{N}$.

Proof Let $R$ and $R^{\prime}$ be the sets of non-trivial rotations and the reflections of $G$ respectively, since $n=\prod_{i=1}^{d} p_{i}^{\alpha_{i}}$, then each element of order $p_{i}^{\alpha}$, where $\alpha$ is the greatest prime power, generates cyclic subgroup of order $p_{i}^{\alpha}-1$. Recall that we have $d$ distinct primes, so there exist total of $d$ complete non-trivial cliques whose each vertex is adjacent to $e$. Let $I$ be the set of the isolated vertices of $\Gamma^{o p p}(G)$, then $I \subset R$ and

$$
|I|=|R|-\left(\sum_{i=1}^{d}\left(p_{i}^{\alpha_{i}}-1\right)\right)
$$




$$
\begin{aligned}
& =(n-1)-p_{1}^{\alpha_{1}}-p_{2}^{\alpha_{2}}-\ldots-p_{i}^{\alpha_{i}}+d \\
& =n+d-\left(1+\sum_{i=1}^{d} p_{i}^{\alpha_{i}}\right) .
\end{aligned}
$$

If $p_{1}=2$, then there exist an element $a \in R \ni a^{2^{\alpha_{1}}}=e$, let $X$ be the set of these $a^{\prime} s$, then $\left\{X, R^{\prime}\right\}$ is a clique of size $n+2^{\alpha_{1}}-1$. We can now see that $\Gamma^{o p p}(G)$ is the union of the non-trivial complete cliques and set $I$, that is,

$$
\begin{aligned}
\Gamma^{o p p}(G) & \left.=K_{1}+\left[\bigcup_{i=1}^{d-1} K_{\left(p_{i}^{\alpha_{i}}-1\right)} \bigcup K_{n+2^{\alpha_{1}}-1}\right)\right] \bigcup I \\
& \left.=K_{1}+\left[\bigcup_{i=1}^{d-1} K_{\left(p_{i}^{\alpha_{i}}-1\right)} \bigcup K_{n+2^{\alpha_{1}}-1}\right)\right] \bigcup \bar{K}_{n+d-\left(1+\sum_{i=1}^{d} p_{i}^{\alpha_{i}}\right)}
\end{aligned}
$$

If $n$ is odd, then $Z(G)$ is trivial and therefore each prime generate distinct clique. So

$$
\begin{aligned}
\Gamma^{o p p}(G) & =K_{1}+\left[\bigcup_{i=1}^{d} K_{\left(p_{i}^{\alpha_{i}}-1\right)} \cup K_{n}\right] \bigcup I \\
& =K_{1}+\left[\bigcup_{i=1}^{d} K_{\left(p_{i}^{\alpha_{i}}-1\right)} \cup K_{n}\right] \bigcup \bar{K}_{n+d-\left(1+\sum_{i=1}^{d} p_{i}^{\alpha_{i}}\right)}
\end{aligned}
$$

Theorem 3. Let $G$ be a cyclic group, $\mathbb{Z}_{n}$, then

$$
\Gamma^{o p p}(G)=\left\{\begin{array}{l}
K_{p^{\alpha}} \text { if } n=p^{\alpha}, \\
K_{1}+\bigcup_{i=1}^{d} K_{\left(p_{i}^{\alpha_{i}}-1\right)} \bigcup \bar{K}_{n+d-\left(1+\sum_{i=1}^{d} p_{i}^{\alpha_{i}}\right)} \text { if } n=\prod_{i=1}^{d} p_{i}^{\alpha_{i}},
\end{array}\right.
$$

where $d$ is the number of prime divisors of $n$ and $\alpha \in \mathbb{N}$.

Proof: If $n=p^{\alpha}$, then $G$ is a p-group, and so for each $x \in G,|x|=p^{t}, 1 \leq t \leq \alpha$, hence all the elements of $G$ form a single clique in $\Gamma^{o p p}(G)$ and $|G|=p^{\alpha}$, therefore $\Gamma^{o p p}(G)=K_{p^{\alpha}}$ Suppose $n=\prod_{i=1}^{d} p_{i}^{\alpha_{i}}$, observe that for all $n, \mathbb{Z}_{n} \cong R$, where $R$ is the set of rotations of dihedral groups. So by Theorem 2,

$$
\begin{aligned}
\Gamma^{o p p}(G) & \cong \Gamma^{o p p}(R) \\
& =K_{1}+\bigcup_{i=1}^{d} K_{\left(p_{i}^{\alpha_{i}}-1\right)} \bigcup \bar{K}_{n+d-\left(1+\sum_{i=1}^{d} p_{i}^{\alpha_{i}}\right)}
\end{aligned}
$$

The connectivity, regularity and completeness of the order product prime graph on dihedral group and cyclic group is given in Theorem 4 and Theorem 5 below; 
Theorem 4. Let $G$ be a dihedral group, $D_{n}$, then $\Gamma^{o p p}(G)$ is regular and complete only if $n=2^{\alpha}$ and connected only if $n=p^{\alpha}, \forall p$.

Proof: We prove the theorem by considering the degree of the group in two cases below. Case 1: If $n=2^{\alpha}$, then $|G|=2^{\alpha+1}$ and so all the elements of $G$ are powers of 2 , they therefore form single clique in $\Gamma^{o p p}(G)$. Therefore $\Gamma^{o p p}(G)$ is complete since for each pair $\left(x_{i}, x_{j}\right) \in G, x_{i} \sim x_{j}, i \neq j$, hence the graph is also connected and regular by the vertex adjacency.

If $n=p^{\alpha}, p \neq 2$, then $|G|=2 p^{\alpha}$ so we only have the elements of order 2 and $p^{\alpha}$ but not $2 p^{\alpha}$ since $G$ is not cyclic. Let $R$ and $R^{\prime}$ be the sets of non-trivial rotations and the reflections of $G$ respectively, then $R$ and $R^{\prime}$ are distinct cliques and $|R|=n-1$ while $\left|R^{\prime}\right|=n$, hence $\operatorname{deg}\left(x_{i}\right)<\operatorname{deg}\left(y_{i}\right) \forall x_{i} \in R, y_{i} \in R^{\prime}$ since $n-1<n$, therefore $\Gamma^{o p p}(G)$ is not regular and also not complete since $x_{i} \nsim y_{i} \forall i$. But $x_{i} \sim e \sim y_{i}$, that is each element of $R$ is reachable from any element of $R^{\prime}$ through $e$, therefore $\Gamma^{o p p}(G)$ is connected.

Case 2: Suppose $n=\prod_{i=1}^{d} p_{i}^{\alpha_{i}}$, then we have total number of $d$ distinct primes and each prime generate a subgroup which is non-trivial complete clique of order $p_{i}^{\alpha_{i}}$. Also there exist some elements of order $\prod_{i=1}^{d} p_{i}^{\alpha_{i}}$ which are the isolated vertices of the graph by definition. Let $g_{i}, g_{j} \in G \ni g_{i}^{p_{i}^{\alpha_{i}}}=e=g_{i}^{\prod_{i=1}^{d} p_{i}^{\alpha_{i}}}$, then

$g_{i} \nsim g_{j}$, therefore $\Gamma^{o p p}(G)$ is not complete and hence not regular. The fact that $g_{i}$ are isolated vertices shows that the graph is not connected.

Theorem 5. Let $G$ be a cyclic group, $\mathbb{Z}_{n}$, then $\Gamma^{o p p}(G)$ is regular, complete and connected only if $n=p^{\alpha}$.

Proof: Suppose $n=p^{\alpha}$, then by Theorem 3, $\Gamma^{o p p}(G)$ is complete and hence regular and connected. On the other hand, if $n=\prod_{i=1}^{d} p_{i}^{\alpha_{i}}$, then there exist $x \in G \ni x_{i}^{\prod_{i=1}^{d} p_{i}^{\alpha_{i}}}=e$, $x$ is therefore an isolated vertex, hence $\Gamma^{o p p}(G)$ is not connected, not complete and not regular by definition since there is another element $y \in G \ni y^{p_{i}^{\alpha_{i}}}=e$, then $\operatorname{deg}(y)<$ $\operatorname{deg}(x)$

The planarity of the order product prime graph on dihedral group and cyclic group is given in Proposition 1 and Proposition 2 below;

Proposition 1. Let $G$ be a dihedral group, $D_{n}$, then $\Gamma^{o p p}(G)$ is planar only if $n=3$

Proof: By Theorem 1, the size of the maximum clique is $n+1$, so the result follows since, the maximum complete subgraph is less than $K_{5}$

Proposition 2. Let $G$ be a cyclic group, $\mathbb{Z}_{n}$, then $\Gamma^{o p p}(G)$ is planar if

$$
n=\left\{\begin{array}{l}
p^{\alpha}<5, \\
\prod_{i=1}^{d} p_{i}^{\alpha_{i}}, p_{i}^{\alpha_{i}}<5 .
\end{array}\right.
$$


Proof: Observe from Theorem 3 and the above hypothesis that, if $n=p^{\alpha}$, then the maximum complete component is less than $K_{5}$ and so $\Gamma^{o p p}(G)$ is planar, since the size of the maximum clique is $|G|=p^{\alpha}$.

Suppose $n=\prod_{i=1}^{d} p_{i}^{\alpha_{i}}$, then still by Theorem 3, the maximum complete component $\ngtr K_{3}$ since $p_{i}^{\alpha_{i}}<5$ and therefore planar.

We start the investigation of the invariants for the order product prime graph. The investigation begins with the diameter of the graph on dihedral group which is given in Propositions 3.

Proposition 3. Let $G$ be a dihedral group, $D_{n}$, then

$$
\operatorname{diam}\left(\Gamma^{o p p}(G)\right)=\left\{\begin{array}{rc}
1 & \text { if } n=2^{\alpha}, \\
2 & \text { if } n=p^{\alpha}, p \neq 2, \\
\infty & \text { if } n=\prod_{i=1}^{d} p_{i}^{\alpha_{i}}, \forall p .
\end{array}\right.
$$

Proof: If $n=2^{\alpha}$, then by Theorem $4, \Gamma^{o p p}(G)$ is complete, and therefore each of its vertices is central, hence $\operatorname{diam}\left(\Gamma^{o p p}(G)\right)=1$.

Suppose $n=p^{\alpha}, p \neq 2$, then recall that by definition, the vertices of $\Gamma^{o p p}(G)$ are the elements of $G$, and $|G|=2 p^{\alpha}$. So pick $x, y \in G, \ni x^{p^{\alpha}}=e=y^{2}$, then the maximum distance between pair of vertices of $\Gamma^{o p p}(G)$ occur between the vertices $x$ and $y$, but $x \sim e \sim y$, that is the distance to reach $x$ from $y$ is 2 , hence $\operatorname{diam}\left(\Gamma^{o p p}(G)\right)=2$.

If $n=\prod_{i=1}^{d} p_{i}^{\alpha_{i}}$, then by Theorem $4, \Gamma^{o p p}(G)$ is disconnected and hence $\operatorname{diam}\left(\Gamma^{o p p}(G)\right)=\infty$

The diameter of the order product prime graph for cyclic groups are given in Proposition 4 .

Proposition 4. Let $G$ be a cyclic group, $\mathbb{Z}_{n}$, then

$$
\operatorname{diam}\left(\Gamma^{o p p}(G)\right)=\left\{\begin{array}{l}
1 \text { if } n=p^{\alpha}, \\
\infty \text { if } n=\prod_{i=1}^{d} p_{i}^{\alpha_{i}} .
\end{array}\right.
$$

Proof: If $n=p^{\alpha}$, then by Theorem $5, \Gamma^{o p p}(G)$ is complete and so each vertex is central, therefore $\operatorname{diam}\left(\Gamma^{o p p}(G)\right)=1$.

If $n=\prod_{i=1}^{d} p_{i}^{\alpha_{i}}$, then still by Theorem $5, \Gamma^{o p p}(G)$ is disconnected and so $\operatorname{diam}\left(\Gamma^{o p p}(G)\right)=\infty$

The girth of the order product prime graph of the dihedral group and the cyclic group is given in Proposition 5 and Proposition 6.

Proposition 5. Let $G$ be the dihedral group, $D_{n}$, then girth $\left(\Gamma^{o p p}(G)\right)=3$, for all $n$. 
Proof: If $n=p^{\alpha}$, then $|G|=2 p^{\alpha}$. Pick any two elements $x, y \in G$,

$\ni x^{p^{\alpha}}=e=y^{2}$, then $x \sim e \sim y$ is a triangle in $\Gamma^{o p p}(G)$, hence

$\operatorname{girth}\left(\Gamma^{o p p}(G)\right)=3$.

If $n=\prod_{i=1}^{d} p_{i}^{\alpha_{i}}$, then $|G|=2 n=2 \prod_{i=1}^{d} p_{i}^{\alpha_{i}}$. Let $l, m \in G, \ni l^{p_{i}^{\alpha_{i}}}=e=m^{2}$, then also $l \sim e \sim m$ is a triangle in $\Gamma^{o p p}(G)$, therefore $\operatorname{girth}\left(\Gamma^{o p p}(G)\right)=3$

Proposition 6. Let $G$ be a cyclic group, $\mathbb{Z}_{n}, n=p^{\alpha}$, then

$$
\operatorname{girth}\left(\Gamma^{o p p}(G)\right)=\left\{\begin{array}{l}
3 \text { if } p>2, \\
\infty \text { if } p=2, \alpha=1 .
\end{array}\right.
$$

Proof: Since $|G|=p^{\alpha}$, then all $g_{1}, g_{2}, \ldots, g_{p^{\alpha}}$ form single clique in $\Gamma^{o p p}(G)$ since $\Gamma^{o p p}(G)$ is complete by Theorem 5 . That is $\Gamma^{o p p}(G)$ contains triangle and hence $\operatorname{girth}\left(\Gamma^{o p p}(G)\right)=3$. If $p=2, \alpha=1$, then $|G|=2$, hence $\Gamma^{o p p}(G)$ is triangle free, infact has no any cycle, therefore $\operatorname{girth}\left(\Gamma^{o p p}(G)\right)=\infty$

The clique number for the order product prime graph on dihedral group and cyclic groups is given in Proposition 7 and Proposition 8.

Proposition 7. Let $G$ be a dihedral group, $D_{n}$, then

$\omega\left(\Gamma^{o p p}(G)\right)=\left\{\begin{array}{l}2 n \quad \text { if } n=2^{\alpha}, \\ (n+1) \quad \text { if } n=p^{\alpha} \text { or } n=\prod_{i=1}^{d} p_{i}^{\alpha_{i}}, \text { where } n \text { is odd in each case, } \\ \left(n+2^{\alpha}\right) \quad \text { if } n=\prod_{i=1}^{d} p^{\alpha_{i}}, \text { where } n \text { is even. }\end{array}\right.$

Proof: If $n=2^{\alpha}$, then by Theorem $1, \Gamma^{o p p}(G)=K_{2 n}$ and is complete by Theorem 4 with $2 n$ vertices, hence $\omega\left(\Gamma^{o p p}(G)\right)=2 n$.

Suppose $n=p^{\alpha}$ or $\prod_{i=1}^{d} p_{i}^{\alpha_{i}}, n$ odd, then by Theorem 2, the size of the maximum clique is $n+1$. Therefore $\omega\left(\Gamma^{o p p}(G)\right)=n+1$.

If $n=\prod_{i=1}^{d} p^{\alpha_{i}}, n$ even, then by Theorem 2 , the size of the maximum clique is $\left(n+2^{\alpha}\right)$, so $\omega\left(\Gamma^{o p p}(G)\right)=\left(n+2^{\alpha}\right)$

Proposition 8. Let $G$ be a cyclic group, $\mathbb{Z}_{n}$, then

$$
\omega\left(\Gamma^{o p p}(G)\right)=\left\{\begin{array}{l}
n \quad \text { if } n=p^{\alpha}, \\
\max _{i}^{\alpha_{i}} \quad \text { if } n=\prod_{i=1}^{d} p_{i}^{\alpha_{i}} .
\end{array}\right.
$$

Proof: If $n=p^{\alpha}$, then $\Gamma^{o p p}(G)$ is complete by Theorem 5 , hence all the elements of $\Gamma^{o p p}(G)$ form a single clique and therefore $\omega\left(\Gamma^{o p p}(G)\right)=|G|=n$.

Suppose $n=\prod_{i=1}^{d} p_{i}^{\alpha_{i}}$. Pick $x, y \in \Gamma^{o p p}(G) \ni x^{p_{1}^{t}}=e=y^{p_{2}^{t}}$ and $h^{\prod_{i=1}^{d}} p_{i}^{\alpha_{i}}=e, 1 \leq t \leq d$, 
then $h$ is an isolated vertex and the maximum clique size is the biggest among $p_{i}^{\alpha_{i}}$, therefore $\omega\left(\Gamma^{o p p}(G)\right)=\max p_{i}^{\alpha_{i}}$.

In Proposition 9 and Proposition 10, we give the independent number of the order product prime graphs for dihedral group and cyclic group.

Proposition 9. Let $G$ be a dihedral group, $D_{n}$, then

$$
\alpha\left(\Gamma^{o p p}(G)\right)=\left\{\begin{array}{c}
1 \text { if } n=2^{\alpha}, \\
2 \text { if } n=p^{\alpha} p \neq 2, \\
n+2 d-\left(1+\sum_{i=1}^{d} p_{i}^{\alpha_{i}}\right) \text { if } n=\prod_{i=1}^{d} p_{i}^{\alpha_{i}} \text { is even, } \\
n+2 d+\sum_{i=1}^{d} p_{i}^{\alpha_{i}} \text { if } n=\prod_{i=1}^{d} p_{i}^{\alpha_{i}} \text { is odd. }
\end{array}\right.
$$

Proof: If $n=2^{\alpha}$, then by Theorem $4, \Gamma^{o p p}(G)$ is complete and so $\alpha\left(\Gamma^{o p p}(G)\right)=1$.

If $n=p^{\alpha}, p \neq 2$, then by Theorem 1 , there are only two non-trivial independent cliques whose each member is adjacent to $e$. Therefore $\alpha\left(\Gamma^{o p p}(G)\right)=2$.

If $n=\prod_{i=1}^{d} p_{i}^{\alpha_{i}}$, then by Theorem 2, the maximum independent set is

$n+2 d-\left(1+\sum_{i=1}^{d} p_{i}^{\alpha_{i}}\right)=\alpha\left(\Gamma^{o p p}(G)\right)$ if $n$ is even and $n+2 d+\sum_{i=1}^{d} p_{i}^{\alpha_{i}}$ if $n$ if $n$ is odd.

Proposition 10. Let $G$ be a cyclic group, $\mathbb{Z}_{n}$, then

$$
\alpha\left(\Gamma^{o p p}(G)\right)=\left\{\begin{array}{l}
1 \text { if } n=p^{\alpha}, \\
n+2 d-\left(1+\sum_{i=1}^{d} p_{i}^{\alpha_{i}}\right) \text { if } n=\prod_{i=1}^{d} p_{i}^{\alpha_{i}} .
\end{array}\right.
$$

Proof: If $n=p^{\alpha}$, then by Theorem $5, \Gamma^{o p p}(G)$ is complete and so all the vertices are central, therefore $\alpha\left(\Gamma^{o p p}(G)\right)=1$.

If $n=\prod_{i=1}^{d} p_{i}^{\alpha_{i}}$, then by Theorem 3 , the size of the maximum independent set is $n+2 d-$ $\left(1+\sum_{i=1}^{d} p_{i}^{\alpha_{i}}\right)=\alpha\left(\Gamma^{o p p}(G)\right)$

The strength for the connectivity of the order product prime graph of dihedral and cyclic groups is determined in Theorem 6 to Theorem 8. This strength has been determined by the vertex-cut of the graphs.

Theorem 6. Let $G$ be a dihedral group, $D_{n}$ or cyclic group, $\mathbb{Z}_{n}$, then $\Gamma^{o p p}(G)$ is 0-connected if $n=\prod_{i=1}^{d} p_{i}^{\alpha_{i}}$

Proof: By Theorem 4 and Theorem $5, \Gamma^{o p p}(G)$ is disconnected, therefore $\Gamma^{o p p}(G)$ is 0 connected 
Theorem 7. Let $G$ be a dihedral group, $D_{p^{\alpha}}$, then $\Gamma^{o p p}(G)$ is $(n-1)$-connected if $n$ is even and 1-connected if $n$ is odd

Proof: If $n$ is even, then by Theorem $4, \Gamma^{o p p}(G)$ is complete, so we have $n-1$ cut-vertices, therefore $\Gamma^{o p p}(G)$ is $(n-1)$-connected.

If $n$ is odd, then still by the Theorem, $\Gamma^{o p p}(G)$ has one vertex -cut which is $K_{1}$ and so $\Gamma^{o p p}(G)$ is 1-connected

Theorem 8. Let $G$ be a cyclic group, $\mathbb{Z}_{n}, n=p^{\alpha}, \alpha \in \mathbb{N}$, then $\Gamma^{o p p}(G)$ is $(n-1)$ connected.

Proof: The result follows, since by Theorem $5, \Gamma^{o p p}(G)$ is complete

The nilpotency of dihedral groups is investigated in Theorem 9 using the vertex-cut of the graph.

Theorem 9. Let $G$ be a dihedral group, $D_{n}$, then $G$ is nilpotent if $k \geq 2$, where $k$ is vertex-cut of $\Gamma^{o p p}(G)$.

Proof: We need to show that if there exist more than 1 vertices of the graph whose all the non-isolated vertices of the graph are adjacent to them then the group is nilpotent.

Suppose $k \geq 2$, then the central vertices of $\Gamma^{o p p}(G)$ are greater or equals to 2 . This is only possible if $Z(G)$ is non-trivial and the non-isolated vertices of the graph are adjacent to $Z(G)$ and $e$ since $G$ is a group. In this case, we can say that $G$ has even degree and so $n=2^{\alpha}$ or $\prod_{i=1}^{d} 2_{i}^{\alpha_{i}}$. If $n=\prod_{i=1}^{d} 2_{i}^{\alpha_{i}}$, then $\Gamma^{o p p}(G)$ has one cut-vertex which is $e$ by Theorem 1. Suppose $n=2^{\alpha}$ then still by Theorem $1, k=2$ and $|G|=2^{\alpha+1}$, so $G$ is a p-group, therefore $G$ is nilpotent

In Theorem 10, we give the result that shows that the order product prime graph of any two groups are isormorphic if the two groups are isomorphic.

Theorem 10. Let $G$ and $H$ be any two groups, such that $G \cong H$, then $\Gamma^{o p p}(G) \cong \Gamma^{o p p}(H)$

Proof: Let $\phi$ be a map between $G$ and $H, \ni \phi: V(G) \longrightarrow V(H)$, define by

$\phi\left(u_{i}\right) \sim \phi\left(u_{j}\right)$ in $H$ if $u_{i} \sim u_{j}$ in $G$, where $u_{i}, v_{j}, 1 \leq i, j \leq|G|$ are the sets of vertices of $G$ respectively. We need to show that $\phi$ is a bijection and preserves vertex adjacency. Pick $u_{1}, u_{2} \in \Gamma^{o p p}(G), \ni u_{1} \sim u_{2}$, then $u_{1} u_{2} \in E\left(\Gamma^{o p p}(G)\right)$ and since $G \cong H$, then $\phi\left(u_{1}\right) \sim \phi\left(u_{2}\right)$ in $H$, that is

$\phi\left(u_{1}\right) \phi\left(u_{2}\right)=v_{1} v_{2} \in E\left(\Gamma^{o p p}(H)\right)$, therefore $\phi$ preserves vertex adjacency. $\phi$ is a bijection since

$$
\begin{aligned}
\phi\left(u_{1}\right) & =\phi\left(u_{2}\right) \Longleftrightarrow u_{1}=u_{2} \\
v_{1} & =v_{2} \Longleftrightarrow u_{1}=v_{1} \text { and } u_{2}=v_{2}
\end{aligned}
$$


and also the vertices $u_{i} \in G \ni \phi\left(u_{i}\right)=v_{i} \in H$, with the condition that $v_{1} v_{2} \in E\left(\Gamma^{o p p}(H)\right)$ if $u_{1} u_{2} \in E\left(\Gamma^{o p p}(G)\right)$ since $G \cong H$

Example 2. Consider the dihedral group of degree six and symmetric group of degree three. Let $G=D_{6}$ and $H=S_{3}$, then $G \cong H$, it can be easily seen that $\Gamma^{o p p}(G) \cong \Gamma^{o p p}(H)$ since the isomorphism preserves the order of the elements in the groups. Observe that the cycles of length two in $S_{3}$ correspond to the reflections of $D_{6}$ while the cycles of length three corresponds to the rotations. Therefore $\Gamma^{o p p}(G) \cong \Gamma^{o p p}(H)$.

\section{Conclusion}

In this paper, new graph that relates the order of the group elements by prime power is defined and some properties of the graph which include some of its invariants, its regularity, planarity, connectivity and the strength of the connectivity have been investigated and the nilpotency of the graph is checked using the vertex-cut of the graph.

\section{Acknowledgements}

The first author would like to thank Federal University of Kashere (FUK) for their total support. He also like to appreciate Universiti Teknologi Malaysia (UTM) for the financial support of International Doctoral Fellowship (IDF). The second and third authors would like to thank Universiti Teknologi Malaysia (UTM) for its support.

\section{References}

[1] Abd Rhani, N., Muhainiah, N. M. A., Sarmin, N. H. and Erfanian, A. On the Domination Number and Regularity of the Relative Coprime Graph of a Group Malaysian Journal of Fundamental and Applied Sciences, 13 (2):(2017), 72-74. doi:10.2307/2369306 2017

[2] Arthur, C. Desiderata and suggestions: No. 2. The Theory of groups: graphical representation American Journal of Mathematics, 1 (2):(1878), 174-6. doi:10.2307/2369306 1878

[3] Akbari, A. and Eza, M. A. Groups for which the Non-Commuting Graph is a Split Graph International Journal of Group Theory 6(1): 29-35 2017

[4] Ganesan, A. On the Automorphism Group of Cayley Graphs Generated by Transposition Australasian Journal of Combinatorics, 64(3): 432-436 2016

[5] Ghorbani, M. and Alkhansari, G. Spectral Properties of NC-graph Kragujevac Journal of Mathematics, 43(4): 523-534 2019

[6] Kakeri, F., Erfanian,A. and Mansoori, F. Generalization of the non-commuting graph of a group via normal subgroup ScienceAsia 42(2016): 231-235 2016 
[7] Kelarev, A., Ryan, j and Yearwood, J. Cayley graphs as classifiers for data mining: The influence of asymmetries. :Discrete Mathematics 2009 309:(2009) 5360-5369.

[8] Selvakumar, K. and Subajini, M. Classification of groups with toroidal coprime graphs. India: Australasian Journal of Combinatorics. 2017. 69(2):(2017), 174-183

[9] Tamizh, T. C., Selvakumar, K. and Raja, S. Commuting Graphs on Dihedral groups The Journal of Mathematics and Computer Science 2011 2(2011):402-406

[10] Sharafdini, R. and Darbandi, r. Energy of Commuting Graphs of finite groups whose Centralizers are abelian arXiv:1704.06464v1/math.co] 2017

[11] Vahidi, j. and Asghar, A. T. The Commuting Graphs on Groups $D_{2 n}, Q_{n}$. The Journal of mathematics and Computer Science 2(2010):123-127 2010 